\title{
Of limited length
}

\author{
Continuously improving precision in length measurements increases understanding of our world and its \\ phenomena, both at small and large scales, as Leo Gross reveals.
}

B ack in the 1860s, Anders Jonas Ångström (pictured) measured the wavelength of visible sunlight with a precision better than $10^{-10} \mathrm{~m}$ by using a spectrometer and proved that the Sun's atmosphere contains hydrogen. When cataloguing the lines in the visible spectrum of sunlight, he introduced a unit length of $10^{-10} \mathrm{~m}$, which was significant in accuracy without the need for fractional numbers. Not only did Ångström's chart and table of wavelengths ${ }^{1}$ become widely used and remained authoritative for decades, his unit for length was adopted and even named after him.

Almost two decades later, Albert Abraham Michelson invented an interferometer that substantially improved the precision of length measurements. This allowed him to define the ångström with respect to an atomic spectroscopic line, namely the red line of cadmium. More importantly, Michelson took advantage of his spectrometer's precision in one of the most central experiments of physics to date: the Michelson-Morley experiment ${ }^{2}$ showed that the speed of light did not change within experimental accuracy, independent of the direction in which the light was travelling, and hence provided strong evidence against the then prevalent ether theory. This was a fundamental result for the special theory of relativity to be developed later by Albert Einstein. The constant speed of light in vacuum has been used since 1983 to define the metre and hence the ångström.

Today, the most accurate length measurements are performed by the Laser Interferometer Gravitational-Wave Observatory (LIGO). Its Michelson interferometers, each arm being $4 \mathrm{~km}$ in length, can detect differences in the average separation of the mirrors on the order of $10^{-18} \mathrm{~m}$. This extreme accuracy enabled the detection of gravitational waves in September 2015: the distances between the mirrors showed a characteristic oscillation that could be traced back to the merger of two black holes ${ }^{3}$.

Maybe counterintuitively, when compared to measuring the separation of the macroscopic mirrors kilometres apart

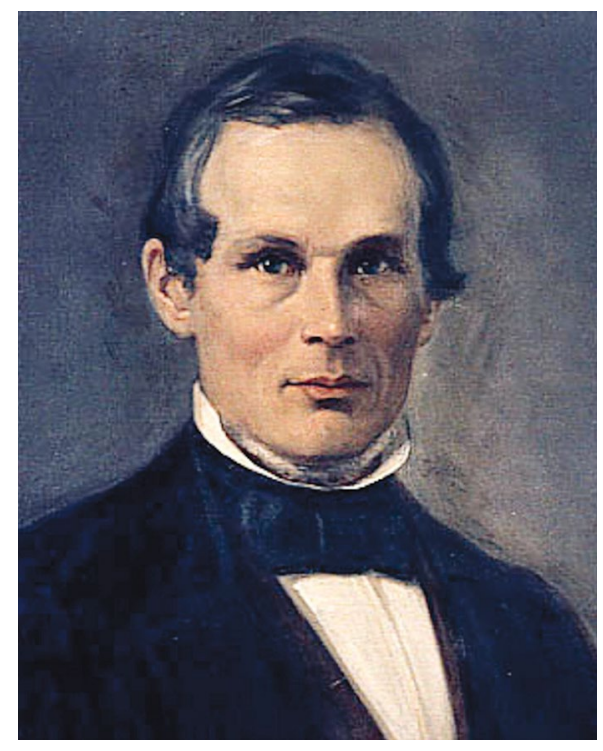

Credit: History and Art Collection / Alamy Stock Photo

in the LIGO detectors, it is more difficult to reach high precision in measuring the length of individual nanoscale objects such as atoms that are about an ångström apart. One reason for this is that in the case of the mirrors one can average over many atoms, whereas when measuring individual atomic distances one cannot. However, by using an atomic force microscope with functionalized probe tips at low temperatures, we can resolve atoms in molecules and discriminate individual bond lengths that differ by only a few picometres ${ }^{4}$ - six orders of magnitude worse than the precision of the LIGO experiment. The manipulation of atoms ${ }^{5}$ enables us to even interact with matter on the ångström scale. Atoms and molecules can be moved, chemical reactions can be induced, and new molecules can be created and studied ${ }^{6}$. In this line of research, speaking about atomic distances in chemistry, crystallography or solidstate physics, the unit ångström is still commonly used, although discouraged by the International Bureau of Weights and Measures for not being an SI unit. But the ångström naturally fits atomic scales as the metre does in our macroscopic world. In addition to its convenient scale for atomic distances, the ångström ties in with the metric system unlike the Bohr radius, sometimes used by theorists, that is not exactly half an ångström but $0.529 \AA$ A long.

To reach such small scales in industrial mass fabrication is certainly even more difficult. Currently, the densest computer chips are produced lithographically in the so-called $7 \mathrm{~nm}$ process node. Next year, the upgrade to the $5 \mathrm{~nm}$ process is foreseen. In a few years - if scaling continues industry might even reach single-digit ångström process nodes. However, these process names do not reflect the dimensions of the produced transistor anymore, but are merely commercial names. In fact, the dimensions of the transistors are larger than the recent process node names. It is still an extremely challenging goal - and not clear if ever reachable - to fabricate devices with ångström precision on an industrial scale.

And in terms of length measurements? What is the limit, and how far will we come? There are still many orders of magnitude to cross until we get to the dimensions of the Planck length, the tiniest length that is physically meaningful, which is on the order of $10^{-35} \mathrm{~m}$. No concepts are in sight to come close to that, but we can be sure that it will be worthwhile to further increase the precision of our measurements and that it will lead to more fundamental insights about our world, the Universe and the laws of physics.

\section{Leo Gross $\mathbb{D}$}

IBM Research - Zurich, Rüschlikon, Switzerland. e-mail: lgr@zurich.ibm.com

\section{Published online: 1 October 2019}

https://doi.org/10.1038/s41567-019-0674-6

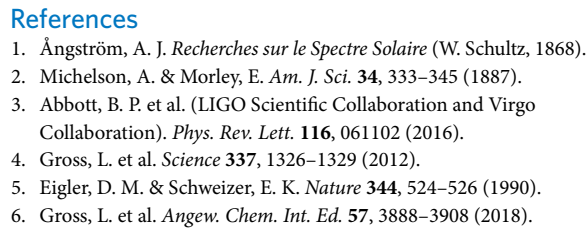

\begin{tabular}{|c|c|c|c|c|c|c|c|c|c|c|c|c|c|c|c|c|}
\hline $\mathrm{m}$ & $e$ & 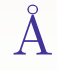 & $\mathrm{S}$ & $\mathrm{u}$ & $R$ & $E_{\mathrm{h}}$ & $F$ & $\Omega$ & ${ }^{\circ} \mathrm{R}$ & $\mu_{0}$ & $\varepsilon_{0}$ & $\alpha$ & $\sigma$ & $\mathrm{V}$ & $\mathrm{R}$ & e \\
\hline
\end{tabular}

